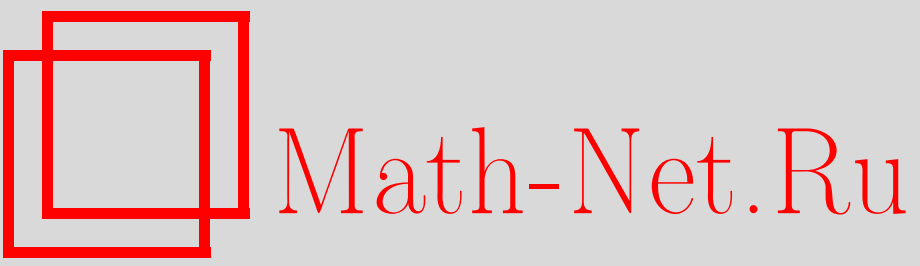

Ю. П. Чубурин, Об операторе Шредингера с малым потенциалом типа возмущенной ступеньки, ТМФ, 1999, том 120, номер 2, 277-290

DOI: https://doi.org/10.4213/tmf775

Использование Общероссийского математического портала Math-Net.Ru подразумевает, что вы прочитали и согласны с пользовательским соглашением

http://www.mathnet.ru/rus/agreement

Параметры загрузки:

IP : 44.207 .124 .84

26 апреля 2023 г., 17:09:17 


\title{
ФИЗИКА
}

Том 120, № 2

август, 1999

(C) 1999 г.

\author{
Ю.П. Чубурин*
}

\section{ОБ ОПЕРАТОРЕ ШРЕДИНГЕРА С МАЛЫМ ПОТЕНЦИАЛОМ ТИПА ВОЗМУШЕННОЙ СТУПЕНЬКИ}

Рассматривается оператор Шредингера с периодическим по двум переменным потенциалом, представляющим собой малую ступеньку, возмушенную убывающей по третьей переменной функцией. Показано, что при определенных условиях на величины ступеньки и возмущения вблизи существенного спектра имеется единственный уровень, который может быть собственным значением или резонансом. Найдено асимптотическое значение этого уровня.

\section{1. ВВЕДЕНИЕ}

Пусть вешественная функция $W(x) \neq 0, x=\left(x_{1}, x_{2}, x_{3}\right) \in \mathbb{R}^{3}$, является периодической по переменным $x_{1}, x_{2}$ с периодом единица и удовлетворяет оценке

$$
|W(x)| \leqslant C \exp \left(-a\left|x_{3}\right|\right), \quad x \in \mathbb{R}^{3},
$$

где $C, a$ - некоторые положительные числа. Обозначим через $\theta\left(x_{3}\right)$ функцию Хевисайда, и пусть $V_{0}<0$. В данной работе рассматриваются самосопряженные операторы $H_{0}\left(k_{\|}\right)=-\Delta+V_{0} \theta\left(x_{3}\right)$ и $H_{\epsilon}\left(k_{\|}\right)=H_{0}\left(k_{\|}\right)+\epsilon W(x), \epsilon>0$, определенные на (достаточно гладких) блоховских по переменным $x_{1}, x_{2}$ функциях из пространства $L^{2}(\Omega)$, где $\Omega=[0,1)^{2} \times \mathbb{R} ; k_{\|}=\left(k_{1}, k_{2}\right) \in \Omega^{*}=[-\pi, \pi)^{2}$ - квазиимпульс. Напомним, что блоховские по $x_{1}, x_{2}$ функции - это сужения на $\Omega$ функций $\phi(x)$, определенных на $\mathbb{R}^{3}$ и удовлетворяющих равенству

$$
\phi\left(x+\left(n_{\|}, 0\right)\right)=e^{\left(k_{\|}, n_{\|}\right)} \phi(x), \quad n_{\|} \in \mathbb{Z}^{2} .
$$

Операторы $H_{0}\left(k_{\|}\right), H_{\epsilon}\left(k_{\|}\right)$можно рассматривать как гамильтонианы электрона вблизи кристаллической поверхности (вид более общего "поверхностного" потенциала указан в статье [1]). Заметим [2-4], что данные операторы порождаются разложением периодических операторов $H_{0}=-\Delta+V_{0} \theta\left(x_{3}\right), H_{\epsilon}=H_{0}+\epsilon W(x)$, действуюших в $L^{2}\left(\mathbb{R}^{3}\right)$, в прямом произведении пространств

$$
\int_{\Omega^{*}}^{\oplus} L^{2}(\Omega) d k_{\|} \simeq L^{2}\left(\Omega \times \Omega^{*}\right)
$$

\footnotetext{
* Физико-технический институт УрО РАН, Ижевск, Россия. E-mail: arg@otf.fti.udmurtia.su
} 
Случай "кристаллической пленки" для малого потеншиала, что соответствует выбору $V_{0}=0$, рассмотрен в статье [5].

Символы $H_{0}\left(k_{\|}\right), H_{\epsilon}\left(k_{\|}\right)$будут также использоваться для обозначения соответствующих дифференциальных выражений. Для $z=\rho e^{i \phi}$, где $\rho>0,-\pi<\phi \leqslant \pi$, полагаем $\sqrt{z}=\sqrt{\rho} e^{i \phi / 2}$.

Под функцией Грина оператора будет пониматься ядро его резольвенты, продолженное, вообше говоря, по параметру $E$ в точки сушественного спектра, а также через вещественную ось на второй лист.

\section{2. ФУНКЦИЯ ГРИНА. ОСНОВНЫЕ УРАВНЕНИЯ}

Лемма 1. Функиия Грина оператора $H_{0}\left(k_{\|}\right)$имеет вид

$$
G_{k_{\|}}\left(x, y, E, V_{0}\right)=\sum_{n_{\|} \in \mathbb{Z}^{2}} \exp \left(i\left(k_{\|}+2 \pi n_{\|}, x_{\|}-y_{\|}\right)\right) G\left(x_{3}, y_{3}, E-\left(k_{\|}+2 \pi n_{\|}\right)^{2}, V_{0}\right),
$$

əде $x_{\|}=\left(x_{1}, x_{2}\right)$ и т.д. Функция Грина оператора $-d^{2} / d x_{3}^{2}+V_{0} \theta\left(x_{3}\right)$ имеет вид

$$
\begin{aligned}
G\left(x_{3}, y_{3}, E, V_{0}\right)=- & \theta\left(x_{3}\right) \theta\left(y_{3}\right)\left[\frac{\exp \left(i \sqrt{E-V_{0}}\left|x_{3}-y_{3}\right|\right)}{2 i \sqrt{E-V_{0}}}+\right. \\
& \left.+\frac{-\sqrt{E}+\sqrt{E-V_{0}}}{2 i \sqrt{E-V_{0}}\left(\sqrt{E}+\sqrt{E-V_{0}}\right)} \exp \left(i \sqrt{E-V_{0}}\left(x_{3}+y_{3}\right)\right)\right]+ \\
& +\theta\left(x_{3}\right) \theta\left(-y_{3}\right) \frac{\exp \left(i \sqrt{E-V_{0}} x_{3}-i \sqrt{E} y_{3}\right)}{i\left(\sqrt{E}+\sqrt{E-V_{0}}\right)}+ \\
& +\theta\left(-x_{3}\right) \theta\left(y_{3}\right) \frac{\exp \left(-i \sqrt{E} x_{3}+i \sqrt{E-V_{0}} y_{3}\right)}{i\left(\sqrt{E}+\sqrt{E-V_{0}}\right)}+ \\
& +\theta\left(-x_{3}\right) \theta\left(-y_{3}\right)\left[\frac{\exp \left(i \sqrt{E}\left|x_{3}-y_{3}\right|\right)}{2 i \sqrt{E}}-\right. \\
& \left.-\frac{-\sqrt{E}+\sqrt{E-V_{0}}}{2 i \sqrt{E}\left(\sqrt{E}+\sqrt{E-V_{0}}\right)} \exp \left(-i \sqrt{E}\left(x_{3}+y_{3}\right)\right)\right] .
\end{aligned}
$$

ДокАЗАТЕльСТво. То, что функция $G\left(x_{3}, y_{3}, E, V_{0}\right)$ является функцией Грина оператора $-d^{2} / d x_{3}^{2}+V_{0} \theta\left(x_{3}\right)$, проверяется непосредственно. Для этого надо учесть, что функция

$$
-\frac{1}{2 i} \sqrt{E} \exp \left(i \sqrt{E}\left|x_{3}-y_{3}\right|\right)
$$

- это функция Грина оператора $-d^{2} / d x_{3}^{2}$. Отсюда без труда можно получить, что функция $G_{k_{\|}}\left(x, y, E, V_{0}\right)$, определяемая формулой (1), является функцией Грина оператора $H_{0}\left(k_{\|}\right)$.

ПРЕДЛОЖЕНИЕ 1. Существенный спектр оператора $H_{\epsilon}\left(k_{\|}\right)$совпадает со спектром оператора $H_{0}\left(k_{\|}\right)$и равен $\left[V_{0}+k_{\|}^{2}, \infty\right)$. 
ДокАЗАтЕльСтво. Спектр оператора $H_{0}\left(k_{\|}\right)$не может быть больше промежутка $\left[V_{0}+k_{\|}^{2}, \infty\right)$, поскольку для $E \notin\left[V_{0}+k_{\|}^{2}, \infty\right)$ оператор, порожденньй функцией Грина, действует в $L^{2}(\Omega)$ (см. вид функции Грина в лемме 1 и рассуждения для случая $V_{0}=0$ в работах $[6,7])$. С другой стороны, для $E>V_{0}+k_{\|}^{2}$, как легко видеть, сушествуют ограниченные решения уравнения $H_{0}\left(k_{\|}\right) \psi=E \psi$. Поэтому спектр оператора $H_{0}\left(k_{\|}\right)$ содержит промежуток $\left[V_{0}+k_{\|}^{2}, \infty\right)[8]$, а значит, в силу сказанного выше совпадает с ним.

Далее, из леммы 1 вытекает, что

$$
W(x) G_{k_{\|}}\left(x, y, i, V_{0}\right) \in L^{2}(\Omega \times \Omega)
$$

(доказательство такое же, как и в случае $\left.V_{0}=0[6,7]\right)$. Утверждение о сушественном спектре следует из теоремы об относительно компактных возмушениях [4].

ПрЕДЛОЖЕНИЕ 2. Предположим, что $E \in \mathbb{R}$, причем $E-\left(k_{\|}+2 \pi n_{\|}\right)^{2} \neq 0 u$ $E-V_{0}-\left(k_{\|}+2 \pi n_{\|}\right)^{2} \neq 0$ для $n_{\|} \in \mathbb{Z}^{2}$. Тогда всякое ограниченное блоховское по $x_{1}, x_{2}$ решение уравнения ІШредингера

$$
H_{\epsilon}\left(k_{\|}\right) \psi=E \psi
$$

удовлетворяет интегральному уравнению

$$
\psi(x)=\psi_{0}(x)-\epsilon \int_{\Omega} G_{k_{\|}}\left(x, y, E, V_{0}\right) W(y) \psi(y) d y
$$

где $\psi_{0}(x)$ - некоторое ограниченное блоховское по $x_{1}, x_{2}$ решение уравнения

$$
H_{0}\left(k_{\|}\right) \psi_{0}=E \psi_{0} .
$$

При этом, если $\psi \in L^{2}\left(\Omega_{0}\right)$, то $\psi_{0}=0$.

Наоборот, если $\psi$ - ограниченное решение уравнения (3) для ограниченного блоховского по $x_{1}, x_{2}$ решения $\psi_{0}$ уравнения (4), то $\psi$ - ограниченное блоховское по $x_{1}, x_{2}$ решение уравнения (2).

ДоКАЗАТЕЛЬСтво. Пусть $\psi$ - решение уравнения (2) из рассматриваемого класса.В силу леммы 1 и условий, наложенных на функции $W, \psi$, интеграл в правой части уравнения (3) существует для $E+i \delta$, где $\delta>0[6,7]$, и определяет блоховскую по переменным $x_{1}, x_{2}$ ограниченную функцию. Применяя к выражению

$$
\psi(x)+\epsilon \int_{\Omega} G_{k_{\|}}\left(x, y, E+i \delta, V_{0}\right) W(y) \psi(y) d y,
$$

где $\delta>0$, оператор $H_{0}\left(k_{\|}\right)-E-i \delta$, пользуясь при этом леммой 1 и переходя затем к пределу $\delta \rightarrow 0$, получаем нуль. Подобными рассуждениями доказывается и обратное утверждение. 
Пусть теперь $\psi \in L^{2}\left(\Omega_{0}\right)$. Устремляя $x_{3} \mathrm{~K}+\infty$ и $-\infty$, получим согласно асимптотике функции $G_{k_{\|}}\left(x, y, E, V_{0}\right)$ (см. лемму 1$)$ следуюшее выражение для функции $\psi_{0}$ :

$$
\begin{aligned}
\psi_{0}(x)= & \sum_{n_{\|} \in \mathbb{Z}^{2}: E-V_{0} \theta\left(x_{3}\right)-\left(k_{\|}+2 \pi n_{\|}\right)^{2}>0} A_{n_{\|}}^{ \pm} \times \\
& \times \exp \left(i\left(\left(k_{\|}+2 \pi n_{\|}, \pm \sqrt{E-V_{0} \theta\left(x_{3}\right)-\left(k_{\|}+2 \pi n_{\|}\right)^{2}}\right), x\right)\right)+\eta(x),
\end{aligned}
$$

где $A^{ \pm}=$const, знак \pm есть $\operatorname{sign} x_{3}, \eta \in L^{2}(\Omega)$. Заметим, что функция

$$
\sum_{n_{\|} \in \mathbb{Z}^{2}: E-V_{0}-\left(k_{\|}+2 \pi n_{\|}\right)^{2}<0} \exp \left(i\left(k_{\|}+2 \pi n_{\|}, x_{\|}-y_{\|}\right)\right) G\left(x_{3}, y_{3}, E-\left(k_{\|}+2 \pi n_{\|}\right)^{2}, V_{0}\right)
$$

не дает вклада в неубываюшие слагаемые; это доказывается, как и в случае $V_{0}=0$ $[6,7]$. С другой стороны, функцию $\psi_{0}$ можно разложить в ряд Фурье по переменным $x_{1}, x_{2}$ :

$$
\psi_{0}(x)=\sum_{n_{\|} \in \mathbb{Z}^{2}} c_{n_{\|}}\left(x_{3}\right) \exp \left(i\left(k_{\|}+2 \pi n_{\|}, x_{\|}\right)\right) .
$$

Очевидно в силу (4), что $c_{n_{\|}}\left(x_{3}\right)$ - ограниченные (как коэффициенты Фурье от ограниченной функции) решения уравнения

$$
-c_{n_{\|}}^{\prime \prime}+V_{0} \theta\left(x_{3}\right) c_{n_{\|}}=\left(E-\left(k_{\|}+2 \pi n_{\|}\right)^{2}\right) c_{n_{\|}} .
$$

Следовательно, сумма (6) конечна, а функции $c_{n_{\|}}\left(x_{3}\right)$ представляют собой при $x_{3}>0 \quad$ и $x_{3}<0$ линейную комбинацию ограниченных экспонент вида $\exp \left( \pm i \sqrt{E-V_{0}-\left(k_{\|}+2 \pi n_{\|}\right)^{2}} x_{3}\right)$ и $\exp \left( \pm i \sqrt{E-\left(k_{\|}+2 \pi n_{\|}\right)^{2}} x_{3}\right)$, соответственно. В соответствии с (5) и ограниченностью экспонент знаки у корней равны sign $x_{3}$. Однако при $V_{0} \neq 0$ гладкая “сшивка" в нуле функций $\exp \left(i \sqrt{E-V_{0}-\left(k_{\|}+2 \pi n_{\|}\right)^{2}} x_{3}\right)$, $x_{3}>0$, и $C \exp \left(-i \sqrt{E-\left(k_{\|}+2 \pi n_{\|}\right)^{2}} x_{3}\right), \quad x_{3}<0$, где $C=$ const, невозможна. Поэтому в ряде (6) имеем $c_{n_{\|}}\left(x_{3}\right)=0$ для всех $n_{\|}$, т.е. $\psi_{0}=0$. Предложение доказано.

В дальнейшем предполагается, что $\operatorname{Im} E \leqslant 0$, причем величина $|\operatorname{Im} E|$ достаточно мала, а также, что

$$
\operatorname{Re} E-V_{0}-\left(k_{\|}+2 \pi n_{\|}\right)^{2}<0, \quad n_{\|} \neq 0,
$$

и величины

$$
k_{3}=\sqrt{E-k_{\|}^{2}}, \quad \kappa_{3}=\sqrt{E-V_{0}-k_{\|}^{2}}
$$

достаточно малы. Заметим, что в случае $E>k_{\|}^{2}$ величину $k_{3}$ можно рассматривать как третью компоненту импульса частицы в пустом полупространстве; малость $\kappa_{3}$ означает малость $V_{0}$.

Пусть в уравнении (3) $\psi_{0}=0$. Отсутствие "налетаюшей волны" и экспоненциальное возрастание при $x_{3} \rightarrow \infty$ для $\operatorname{Re} E>V_{0}+k_{\|}^{2}$, а для $\operatorname{Re} E>k_{\|}^{2}$ и при $x_{3} \rightarrow-\infty$ 
нетривиальных решений $\psi$ такого уравнения для $E$ на втором листе с $\operatorname{Im} E<0$ (экспоненциальное возрастание вытекает из вида функции Грина) позволяют рассматривать это уравнение для данных $E$ как уравнение для определения резонансов [9].

Итак, с помошью уравнения (3) при $\psi_{0}=0$ определяются как собственные, так и резонансные уровни. Запишем уравнение $(3)$ с $\psi_{0}=0$ для новой неизвестной функции $\phi(x)=\sqrt{\epsilon|W(x)|} \psi(x)[10]$ и $E \neq V_{0}+k_{\|}^{2}, E \neq k_{\|}^{2}$ в виде

$$
\phi(x)=-\epsilon \int_{\Omega} \sqrt{|W(x)|} G_{\|}\left(x, y, E, V_{0}\right) \sqrt{W(y)} \phi(y) d y
$$

где $\sqrt{W(x)}=\operatorname{sign} W(x) \sqrt{|W(x)|}$. Перейдем от величин $E, V_{0}$ в выражении для функции Грина к величинам $k_{3}, \kappa_{3}$, определяемым равенствами (7). При этом будем пользоваться обозначениями типа $G_{k_{\|}}\left(x, y, k_{3}, \kappa_{3}\right)$. Введем также обозначение

$G_{\|}^{(1)}\left(x, y, k_{3}, \kappa_{3}\right)=\sum_{n_{\|} \in \mathbb{Z}^{2} \backslash\{(0,0)\}} \exp \left(i\left(k_{\|}+2 \pi n_{\|}, x_{\|}-y_{\|}\right)\right) G\left(x_{3}, y_{3}, E-\left(k_{\|}+2 \pi n_{\|}\right)^{2}, V_{0}\right)$.

ПРЕДЛОЖЕНИЕ 3 . Пусть $k_{\|}$не принадлежит границе $\Omega^{*}\left(\right.$ в $\left.\mathbb{R}^{2}\right)$. Имеет место равенство

$$
\begin{aligned}
G_{k_{\|}}(x, y, & \left.k_{3}, \kappa_{3}\right)=-\frac{\exp \left(i\left(k_{\|}, x_{\|}-y_{\|}\right)\right)}{i\left(k_{3}+\kappa_{3}\right)}\left[\theta\left(x_{3}\right) \theta\left(y_{3}\right) \exp \left(i \kappa_{3}\left(x_{3}+y_{3}\right)\right)+\right. \\
& +\theta\left(x_{3}\right) \theta\left(-y_{3}\right) \exp \left(i \kappa_{3} x_{3}-i k_{3} y_{3}\right)+\theta\left(-x_{3}\right) \theta\left(y_{3}\right) \exp \left(-i k_{3} x_{3}+i \kappa_{3} y_{3}\right)+ \\
& \left.+\theta\left(-x_{3}\right) \theta\left(-y_{3}\right) \exp \left(-i k_{3}\left(x_{3}+y_{3}\right)\right)\right]+G_{k_{\|}}^{(2)}\left(x, y, k_{3}, \kappa_{3}\right)
\end{aligned}
$$

где функиия $G_{k_{\|}}^{(2)}$ обладает тем свойством, что

$$
\sqrt{|W(x)|} G_{k_{\|}}^{(2)}\left(x, y, k_{3}, \kappa_{3}\right) \sqrt{W(y)}
$$

представляет собой аналитическую $L^{2}(\Omega \times \Omega)$-значную функцию переменных $k_{3}, \kappa_{3}$ в некоторой комплексной окрестности $U$ точки $(0,0) \in \mathbb{C}^{2}$.

ДоКАЗАТЕЛЬСТво. Используя лемму 1, получаем

$$
\begin{aligned}
G_{k_{\|}}\left(x, y, k_{3}, \kappa_{3}\right)=-\frac{\exp \left(i\left(k_{\|}, x_{\|}-y_{\|}\right)\right)}{i\left(k_{3}+\kappa_{3}\right)}\left[\theta\left(x_{3}\right) \theta\left(y_{3}\right) \exp \left(i \kappa_{3}\left(x_{3}+y_{3}\right)\right)+\right. \\
\quad+\theta\left(x_{3}\right) \theta\left(-y_{3}\right) \exp \left(i \kappa_{3} x_{3}-i k_{3} y_{3}\right)+\theta\left(-x_{3}\right) \theta\left(y_{3}\right) \exp \left(-i k_{3} x_{3}+i \kappa_{3} y_{3}\right)+ \\
\left.\quad+\theta\left(-x_{3}\right) \theta\left(-y_{3}\right) \exp \left(-i k_{3}\left(x_{3}+y_{3}\right)\right)\right]- \\
\quad-\exp \left(i\left(k_{\|}, x_{\|}-y_{\|}\right)\right)\left[\theta\left(x_{3}\right) \theta\left(y_{3}\right) \frac{\exp \left(i \kappa_{3}\left|x_{3}-y_{3}\right|\right)-\exp \left(i \kappa_{3}\left(x_{3}+y_{3}\right)\right)}{2 i \kappa_{3}}+\right. \\
\left.\quad+\theta\left(-x_{3}\right) \theta\left(-y_{3}\right) \frac{\exp \left(i k_{3}\left|x_{3}-y_{3}\right|\right)-\exp \left(i k_{3}\left(x_{3}+y_{3}\right)\right)}{2 i k_{3}}\right]+G_{k_{\|}}^{(1)}\left(x, y, k_{3}, \kappa_{3}\right)
\end{aligned}
$$


Обозначим через $G_{k_{\|}}^{(2)}$ сумму двух последних слагаемых в полученном выражении. Для первого из этих слагаемых свойство, которым должна обладать функция $G_{k_{\|}}^{(2)}$, очевидно. Для функции $G_{k_{\|}}^{(1)}$ это свойство доказьвается с учетом сделанных предположений и того обстоятельства, что в силу условия на $k_{\|}$в формулировке теоремы знаменатели у слагаемых, входящих в состав функции $G_{k_{\|}}^{(1)}$, не обрашаются в нуль. Данное доказательство вполне аналогично случаю $V_{0}=0[6,7]$. Предложение доказано.

Обозначим через $K_{k_{\|}}\left(k_{3}, \kappa_{3}\right)$ оператор с ядром $-\sqrt{|W(x)|} G_{k_{\|}}^{(2)}\left(x, y, k_{3}, \kappa_{3}\right) \sqrt{W(y)}$. Введем в рассмотрение для малых $\epsilon$ следуюшие величины:

$$
\begin{aligned}
\alpha_{k_{\|}}^{(1,1)}\left(k_{3}, \kappa_{3}\right)= & \epsilon \int_{\Omega} \exp \left(-i\left(\left(k_{\|},-\kappa_{3}\right), y\right)\right) \theta\left(y_{3}\right) \sqrt{W(y)}\left(1-\epsilon K_{k_{\|}}\left(k_{3}, \kappa_{3}\right)\right)^{-1} \times \\
& \times\left(\theta\left(y_{3}\right) \sqrt{|W(y)|} \exp \left(i\left(\left(k_{\|}, \kappa_{3}\right), y\right)\right)\right) d y, \\
\alpha_{k_{\|}}^{(2,1)}\left(k_{3}, \kappa_{3}\right)= & \epsilon \int_{\Omega} \exp (-i(k, y)) \theta\left(-y_{3}\right) \sqrt{W(y)}\left(1-\epsilon K_{k_{\|}}\left(k_{3}, \kappa_{3}\right)\right)^{-1} \times \\
& \times\left(\theta\left(y_{3}\right) \sqrt{|W(y)|} \exp \left(i\left(\left(k_{\|}, \kappa_{3}\right), y\right)\right)\right) d y, \\
\alpha_{k_{\|}}^{(1,2)}\left(k_{3}, \kappa_{3}\right)= & \epsilon \int_{\Omega} \exp \left(-i\left(\left(k_{\|},-\kappa_{3}\right), y\right)\right) \theta\left(y_{3}\right) \sqrt{W(y)}\left(1-\epsilon K_{k_{\|}}\left(k_{3}, \kappa_{3}\right)\right)^{-1} \times \\
& \times\left(\theta\left(-y_{3}\right) \sqrt{|W(y)|} \exp \left(i\left(\left(k_{\|},-k_{3}\right), y\right)\right)\right) d y, \\
\alpha_{k_{\|}}^{(2,2)}\left(k_{3}, \kappa_{3}\right)= & \epsilon \int_{\Omega} \exp (-i(k, y)) \theta\left(-y_{3}\right) \sqrt{W(y)}\left(1-\epsilon K_{k_{\|}}\left(k_{3}, \kappa_{3}\right)\right)^{-1} \times \\
& \times\left(\theta\left(-y_{3}\right) \sqrt{|W(y)|} \exp \left(i\left(\left(k_{\|},-k_{3}\right), y\right)\right)\right) d y .
\end{aligned}
$$

Для достаточно малых $\epsilon$ получаем, пользуясь разложением операторнозначной функции $\left(1-\epsilon K_{k_{\|}}\left(k_{3}, \kappa_{3}\right)\right)^{-1}$ в ряд по степеням $\epsilon$, следуюшие соотношения:

$$
\begin{aligned}
& \alpha_{k_{\|}}^{(1,1)}\left(k_{3}, \kappa_{3}\right)=\epsilon \int_{\Omega} \exp \left(2 i \kappa_{3} y_{3}\right) \theta\left(y_{3}\right) W(y) d y+\beta_{k_{\|}}^{(1,1)}\left(k_{3}, \kappa_{3}\right), \\
& \alpha_{k_{\|}}^{(2,1)}\left(k_{3}, \kappa_{3}\right)=\beta_{k_{\|}}^{(2,1)}\left(k_{3}, \kappa_{3}\right), \\
& \alpha_{k_{\|}}^{(1,2)}\left(k_{3}, \kappa_{3}\right)=\beta_{k_{\|}}^{(1,2)}\left(k_{3}, \kappa_{3}\right), \\
& \alpha_{k_{\|}}^{(2,2)}\left(k_{3}, \kappa_{3}\right)=\epsilon \int_{\Omega} \exp \left(-2 i k_{3} y_{3}\right) \theta\left(-y_{3}\right) W(y) d y+\beta_{k_{\|}}^{(2,2)}\left(k_{3}, \kappa_{3}\right),
\end{aligned}
$$

где

$$
\begin{aligned}
\beta_{k_{\|}}^{(1,1)}\left(k_{3}, \kappa_{3}\right)= & \epsilon \sum_{n=1}^{\infty} \epsilon^{n} \int_{\Omega} \exp \left(-i\left(\left(k_{\|},-\kappa_{3}\right), y\right)\right) \theta\left(y_{3}\right) \sqrt{W(y)} \times \\
& \times\left(K_{k_{\|}}\left(k_{3}, \kappa_{3}\right)\right)^{n}\left(\theta\left(y_{3}\right) \sqrt{|W(y)|} \exp \left(i\left(\left(k_{\|}, \kappa_{3}\right), y\right)\right)\right) d y \\
\beta_{k_{\|}}^{(2,1)}\left(k_{3}, \kappa_{3}\right)= & \epsilon \sum_{n=1}^{\infty} \epsilon^{n} \int_{\Omega} \exp (-i(k, y)) \theta\left(-y_{3}\right) \sqrt{W(y)} \times
\end{aligned}
$$




$$
\begin{aligned}
& \times\left(K_{k_{\|}}\left(k_{3}, \kappa_{3}\right)\right)^{n}\left(\theta\left(y_{3}\right) \sqrt{|W(y)|} \exp \left(i\left(\left(k_{\|}, \kappa_{3}\right), y\right)\right)\right) d y \\
\beta_{k_{\|}}^{(1,2)}\left(k_{3}, \kappa_{3}\right)= & \epsilon \sum_{n=1}^{\infty} \epsilon^{n} \int_{\Omega} \exp \left(-i\left(\left(k_{\|},-\kappa_{3}\right), y\right)\right) \theta\left(y_{3}\right) \sqrt{W(y)} \times \\
& \times\left(K_{k_{\|}}\left(k_{3}, \kappa_{3}\right)\right)^{n}\left(\theta\left(-y_{3}\right) \sqrt{|W(y)|} \exp \left(i\left(\left(k_{\|},-k_{3}\right), y\right)\right)\right) d y \\
\beta_{k_{\|}}^{(2,2)}\left(k_{3}, \kappa_{3}\right)= & \epsilon \sum_{n=1}^{\infty} \epsilon^{n} \int_{\Omega} \exp (-i(k, y)) \theta\left(-y_{3}\right) \sqrt{W(y)} \times \\
& \times\left(K_{k_{\|}}\left(k_{3}, \kappa_{3}\right)\right)^{n}\left(\theta\left(-y_{3}\right) \sqrt{|W(y)|} \exp \left(i\left(\left(k_{\|},-k_{3}\right), y\right)\right)\right) d y .
\end{aligned}
$$

В соответствии с предложением 3 и теоремой Вейерштрасса об аналитичности равномерно сходяшихся рядов из аналитических функций функции $\alpha_{k_{\|}}^{(i, j)}\left(k_{3}, \kappa_{3}\right)$ и $\beta_{k_{\|}}^{(i, j)}\left(k_{3}, \kappa_{3}\right)$ $(i, j=1,2)$ аналитически зависят от $\left(k_{3}, \kappa_{3}\right)$ в некоторой комплексной окрестности нуля $\mathcal{V} \subseteq \mathbb{C}^{2}$. При этом имеет место оценка

$$
\left|\beta_{k_{\|}}^{(i, j)}\left(k_{3}, \kappa_{3}\right)\right| \leqslant C \epsilon^{2}
$$

где константу $C$ можно считать не зависящей от $\left(k_{3}, \kappa_{3}\right) \in \mathcal{V}$.

Положим

$$
\begin{gathered}
f_{1}\left(\kappa_{3}\right)=\int_{\Omega} \exp \left(2 i \kappa_{3} y_{3}\right) \theta\left(y_{3}\right) W(y) d y \\
f_{2}\left(k_{3}\right)=\int_{\Omega} \exp \left(-2 i k_{3} y_{3}\right) \theta\left(-y_{3}\right) W(y) d y \\
f\left(k_{3}, \kappa_{3}\right)=f_{1}\left(\kappa_{3}\right)+f_{2}\left(k_{3}\right), \\
\beta_{k_{\|}}^{(1)}=\beta_{k_{\|}}^{(1,1)}+\beta_{k_{\|}}^{(2,1)}, \quad \beta_{k_{\|}}^{(2)}=\beta_{k_{\|}}^{(1,2)}+\beta_{k_{\|}}^{(2,2)} \\
\beta_{k_{\|}}=\frac{1}{i}\left(\beta_{k_{\|}}^{(1)}+\beta_{k_{\|}}^{(2)}\right) .
\end{gathered}
$$

ЛЕмма 2. Предположим, что

$$
f(0,0)=\int_{\Omega} W(x) d x \neq 0 .
$$

Пусть для соответствующих величинам $E$ и $V_{0}$ величин $k_{3}$ и $\kappa_{3}$ имеет место включение $\left(k_{3}, \kappa_{3}\right) \in \mathcal{V}$. Тогде уравнение (8) обладает ненулевым решением в классе $L^{2}(\Omega)$ для достаточно мальх є в том и только в том случае, если выполнено равенство

$$
k_{3}+\kappa_{3}=\frac{\epsilon}{i} f\left(k_{3}, \kappa_{3}\right)+\beta_{k_{\|}}\left(k_{3}, \kappa_{3}\right)
$$

При этом решение уравнения (8) единственно с точностью до числового множителя. 
ДОКАЗАТЕЛЬСТво (ср. с [5]). Положим

$$
\theta(x)=\left(1-\epsilon K_{k_{\|}}\left(k_{3}, \kappa_{3}\right)\right) \phi(x),
$$

тогда уравнение (8) с учетом предложения 3 можно записать в виде

$$
\begin{aligned}
\theta(x)= & \frac{\epsilon \sqrt{|W(x)|}}{i\left(k_{3}+\kappa_{3}\right)} e^{i\left(k_{\|}, x_{\|}\right)} \int_{\Omega} e^{-i\left(k_{\|}, y_{\|}\right)} \times \\
& \times\left[\theta\left(x_{3}\right) \theta\left(y_{3}\right) \exp \left(i \kappa_{3}\left(x_{3}+y_{3}\right)\right)+\theta\left(x_{3}\right) \theta\left(-y_{3}\right) \exp \left(i \kappa_{3} x_{3}-i k_{3} y_{3}\right)+\right. \\
& \left.+\theta\left(-x_{3}\right) \theta\left(y_{3}\right) \exp \left(-i k_{3} x_{3}+i \kappa_{3} y_{3}\right)+\theta\left(-x_{3}\right) \theta\left(-y_{3}\right) \exp \left(-i k_{3}\left(x_{3}+y_{3}\right)\right)\right] \times \\
& \times \sqrt{W(y)}\left(1-\epsilon K_{k_{\|}}\left(k_{3}, \kappa_{3}\right)\right)^{-1} \theta(y) d y
\end{aligned}
$$

откуда

$$
\begin{aligned}
\theta(x)= & C_{1} \theta\left(x_{3}\right) \sqrt{|W(x)|} \exp \left(i\left(\left(k_{\|}, \kappa_{3}\right), x\right)\right)+ \\
& +C_{2} \theta\left(-x_{3}\right) \sqrt{|W(x)|} \exp \left(i\left(\left(k_{\|},-k_{3}\right), x\right)\right),
\end{aligned}
$$

где $C_{1}, C_{2}$ - некоторые константы. Подставляя выражение (16) для функции $\theta\left(x_{3}\right)$ в $(15)$ и полагая последовательно $x_{3}>0$ и $x_{3}<0$, получаем с учетом соотношений $(9)-(11)$, (13) равенства

$$
\begin{aligned}
& C_{1}=\frac{1}{i\left(k_{3}+\kappa_{3}\right)}\left[C_{1}\left(\epsilon f_{1}\left(\kappa_{3}\right)+\beta_{k_{\|}}^{(1)}\left(k_{3}, \kappa_{3}\right)\right)+C_{2}\left(\epsilon f_{2}\left(k_{3}\right)+\beta_{k_{\|}}^{(2)}\left(k_{3}, \kappa_{3}\right)\right)\right], \\
& C_{2}=\frac{1}{i\left(k_{3}+\kappa_{3}\right)}\left[C_{1}\left(\epsilon f_{1}\left(\kappa_{3}\right)+\beta_{k_{\|}}^{(1)}\left(k_{3}, \kappa_{3}\right)\right)+C_{2}\left(\epsilon f_{2}\left(k_{3}\right)+\beta_{k_{\|}}^{(2)}\left(k_{3}, \kappa_{3}\right)\right)\right] .
\end{aligned}
$$

Предположим, что $\theta\left( \pm x_{3}\right) W(x) \neq 0$ (случаи $\theta\left(x_{3}\right) W(x)=0$ или $\theta\left(-x_{3}\right) W(x)=0$ рассматриваются аналогично с соответствуюшими упрощениями). Тогда условие существования ненулевого решения уравнения (8), очевидно, эквивалентно сушествованию ненулевого решения $\left(C_{1}, C_{2}\right)$ полученной однородной системы линейных уравнений $(17)$, (18) или равенству нулю определителя ее матрицы

$$
-\frac{1}{\left(k_{3}+\kappa_{3}\right)^{2}}\left[-\left(k_{3}+\kappa_{3}\right)^{2}-i\left(k_{3}+\kappa_{3}\right)\left(\epsilon f\left(k_{3}, \kappa_{3}\right)+i \beta_{k_{\|}}\left(k_{3}, \kappa_{3}\right)\right)\right]=0,
$$

откуда сразу вытекает равенство (14). Единственность решения уравнения (8) с точностью до множителя следует из соотношения (16) и того факта, что ранг матрицы системы уравнений $(17),(18)$ равен единище. Действительно, из условия $f(0,0) \neq 0$ вытекает, что одно из чисел $f_{1}(0), f_{2}(0)$ отлично от нуля. Пусть, например, $f_{1}(0) \neq 0$. Тогда для малых $\kappa_{3}$ имеем $f_{1}\left(\kappa_{3}\right) \neq 0$ и элемент матришы $\epsilon f_{1}\left(\kappa_{3}\right)+\beta_{k_{\|}}^{(1)}\left(k_{3}, \kappa_{3}\right)=\epsilon f_{1}\left(\kappa_{3}\right)+o(\epsilon)$ (см. (12)) при малых $\epsilon$ не равен нулю. Тем самым лемма доказана.

СлЕДСтвиЕ. В случае существования для всех $\epsilon>0$ решения уравнения (8) при условии ограниченности величины $V_{0}=V_{0}(\epsilon)$ должно выполняться соотночение $V_{0}=O(\epsilon)$.

ДокАЗАТЕЛЬСТво СЛЕДСтвия. В силу соотношений (14), (7) и (12) имеем $\sqrt{E-k_{\|}^{2}}+\sqrt{E-V_{0}-k_{\|}^{2}}=O(\epsilon)$, откуда $V_{0}=O(\epsilon)$. 


\section{3. АСИМПТОТИКА УРОВНЕЙ}

Положим $V_{0}=-A \epsilon^{\sigma}$, где $A>0, \sigma \geqslant 1$ - некоторые константы. Пусть вначале $\sigma>2$. Положим $\nu=\min (\sigma-1,2)$ и выберем числа $K>0$ и $\delta \in(1, \nu)$. Далее будем предполагать, что

$$
f(0,0)=\int_{\Omega} W(x) d x \neq 0 .
$$

Теорема 1. Уравнение (8) при всех достаточно малых $\epsilon$ для совокупности чисел $E$, удовлетворяющих неравенству

$$
\left|\sqrt{E-k_{\|}^{2}}-\frac{\epsilon}{2 i} \int_{\Omega} W(x) d x\right| \leqslant K \epsilon^{\delta},
$$

имеет единственное с точностью до мнохителя ненулевое решение в классе $L^{2}(\Omega)$. Для соответствующего решения Е справедливо соотношение

$$
E=k_{\|}^{2}-\frac{\epsilon^{2}}{4}\left(\int_{\Omega} W(x) d x\right)^{2}+O\left(\epsilon^{1+\nu}\right) .
$$

ДокАЗАТЕЛЬство. Перепишем равенство (14) в виде

$$
k_{3}=-\sqrt{k_{3}^{2}+A \epsilon^{\sigma}}+\frac{\epsilon}{i} f\left(k_{3}, \sqrt{k_{3}^{2}+A \epsilon^{\sigma}}\right)+\beta_{k_{\|}}\left(k_{3}, \epsilon\right) .
$$

Будем рассматривать равенство (22) как уравнение относительно $k_{3}$ в множестве вида (20). Множество (20) запишем для $k_{3}$ в виде

$$
S_{\epsilon}=\left\{k_{3} \in \mathbb{C}:\left|k_{3}-\frac{\epsilon}{2 i} f(0,0)\right| \leqslant K \epsilon^{\delta}\right\}
$$

Заметим, что для малых $\epsilon$ в силу условия (19) следует $0 \notin S_{\epsilon}$.

Имеем для $k_{3} \in S_{\epsilon}$ равенство

$$
\sqrt{k_{3}^{2}+A \epsilon^{\sigma}}=k_{3} \sqrt{1+\frac{A \epsilon^{\sigma}}{k_{3}^{2}}}=k_{3}\left[1+\frac{A \epsilon^{\sigma}}{2 k_{3}^{2}}+O\left(\left(\frac{\epsilon^{\sigma}}{k_{3}^{2}}\right)^{2}\right)\right] .
$$

Из того, что $k_{3} \in S_{\epsilon}$, вытекают соотношения $k_{3}=O(\epsilon)$ и $k_{3}^{-2}=O\left(\epsilon^{-2}\right)(\epsilon$ мало). Используя эти соотношения, запишем равенство (23) в виде

$$
\sqrt{k_{3}^{2}+A \epsilon^{\sigma}}=k_{3}-a\left(k_{3}, \epsilon\right)
$$

где функция

$$
a\left(k_{3}, \epsilon\right)=-k_{3}\left[\frac{A \epsilon^{\sigma}}{2 k_{3}^{2}}+O\left(\left(\frac{\epsilon^{\sigma}}{k_{3}^{2}}\right)^{2}\right)\right]
$$

удовлетворяет оценке $\left|a\left(k_{3}, \epsilon\right)\right| \leqslant C \epsilon^{\sigma-1}$, причем константа $C$ не зависит от $k_{3} \in S_{\epsilon}$. Далее, в силу аналитичности функций $f_{1}, f_{2}$ и соотношения (24) имеем

$$
\frac{\epsilon}{i} f\left(k_{3}, \sqrt{k_{3}^{2}+A \epsilon^{\sigma}}\right)=\frac{\epsilon}{i} f(0,0)+b\left(k_{3}, \epsilon\right),
$$


где функция $b\left(k_{3}, \epsilon\right)$ удовлетворяет оценке $\left|b\left(k_{3}, \epsilon\right)\right| \leqslant C^{\prime} \epsilon^{2}$, причем $C^{\prime}$ не зависит от $k_{3} \in S_{\epsilon}$. Такая же оценка в силу (12), (13) справедлива для функции $\beta_{k_{\|}}$. Положим

$$
c\left(k_{3}, \epsilon\right)=\frac{1}{2}\left(a\left(k_{3}, \epsilon\right)+b\left(k_{3}, \epsilon\right)+\beta_{k_{\|}}\left(k_{3}, \epsilon\right)\right) .
$$

Вследствие соотношений (24), (25) равенство (22) можно переписать в виде

$$
k_{3}=\frac{1}{2}\left(k_{3}-\sqrt{k_{3}^{2}+A \epsilon^{\sigma}}+\frac{\epsilon}{i} f\left(k_{3}, \sqrt{k_{3}^{2}+A \epsilon^{\sigma}}\right)+\beta_{k_{\|}}\left(k_{3}, \epsilon\right)\right)=\frac{\epsilon}{2 i} f(0,0)+c\left(k_{3}, \epsilon\right),
$$

причем $\left|c\left(k_{3}, \epsilon\right)\right| \leqslant C^{\prime \prime} \epsilon^{\nu}$, где $C^{\prime \prime}$ не зависит от $k_{3} \in S_{\epsilon}$.

Равенство (26) можно рассматривать как уравнение для неподвижной точки отображения (функции)

$$
\begin{aligned}
F\left(k_{3}\right) & =\frac{1}{2}\left(k_{3}-\sqrt{k_{3}^{2}+A \epsilon^{\sigma}}+\frac{\epsilon}{i} f\left(k_{3}, \sqrt{k_{3}^{2}+A \epsilon^{\sigma}}\right)+\beta_{k_{\|}}\left(k_{3}, \epsilon\right)\right)= \\
& =\frac{\epsilon}{2 i} f(0,0)+c\left(k_{3}, \epsilon\right) .
\end{aligned}
$$

Докажем, что отображение $F$ переводит множество $S_{\epsilon}$ в себя и является сжимающим на данном множестве для всех достаточно малых $\epsilon$. Пусть $k_{3} \in S_{\epsilon}$. Тогда в силу сказанного выше

$$
\left|F\left(k_{3}\right)-\frac{\epsilon}{2 i} f(0,0)\right|=\left|c\left(k_{3}, \epsilon\right)\right| \leqslant C \epsilon^{\nu} \leqslant K \epsilon^{\delta}
$$

для достаточно малых $\epsilon$, поскольку $\nu>\delta$. Отсюда следует, что $F\left(k_{3}\right) \in S_{\epsilon}$. Докажем, что $F$ - сжимаюшее отображение. Пусть $k_{3}, \tilde{k}_{3} \in S_{\epsilon}$. Используя формулу $(27)$, имеем

$$
\begin{aligned}
\left|F\left(k_{3}\right)-F\left(\tilde{k}_{3}\right)\right| \leqslant & \frac{1}{2}\left|\left(k_{3}-\sqrt{k_{3}^{2}+A \epsilon^{\sigma}}\right)-\left(\tilde{k}_{3}-\sqrt{\tilde{k}_{3}^{2}+A \epsilon^{\sigma}}\right)\right|+ \\
& +\frac{\epsilon}{2}\left|f\left(k_{3}, \sqrt{k_{3}^{2}+A \epsilon^{\sigma}}\right)-f\left(\tilde{k}_{3}, \sqrt{\tilde{k}_{3}^{2}+A \epsilon^{\sigma}}\right)\right|+ \\
& +\frac{1}{2}\left|\beta_{k_{\|}}\left(k_{3}, \epsilon\right)-\beta_{k_{\|}}\left(\tilde{k}_{3}, \epsilon\right)\right| .
\end{aligned}
$$

Из соотношения $(24)$ и равенства

$$
k_{3}=\frac{\epsilon}{2 i} f(0,0)+O\left(\epsilon^{\delta}\right),
$$

вытекающего из определения множества $S_{\epsilon}$, следует, что

$$
\begin{aligned}
\sqrt{k_{3}^{2}+A \epsilon^{\sigma}}-\sqrt{\tilde{k}_{3}^{2}+A \epsilon^{\sigma}} & =\frac{k_{3}^{2}-\tilde{k}_{3}^{2}}{\sqrt{k_{3}^{2}+A \epsilon^{\sigma}}+\sqrt{\tilde{k}_{3}^{2}+A \epsilon^{\sigma}}}= \\
& =\left(k_{3}-\tilde{k}_{3}\right) \frac{f(0,0)+O\left(\epsilon^{\delta-1}\right)}{f(0,0)+O\left(\epsilon^{\delta-1}\right)+O\left(\epsilon^{\sigma-2}\right)} .
\end{aligned}
$$

В силу соотношений $\delta>1, \sigma>2$ и условия (19) полученное выражение (29) по модулю не превосходит $C_{1}\left|k_{3}-\tilde{k}_{3}\right|$, где $C_{1}=$ const не зависит от (малых) $\epsilon$ и $k_{3}, \tilde{k}_{3} \in S_{\epsilon}$. 
Из (29) следует, что

$$
\left|\left(k_{3}-\sqrt{k_{3}^{2}+A \epsilon^{\sigma}}\right)-\left(\tilde{k}_{3}-\sqrt{\tilde{k}_{3}^{2}+A \epsilon^{\sigma}}\right)\right|=\left|k_{3}-\tilde{k}_{3}\right| O\left(\epsilon^{\mu}\right),
$$

где $\mu=\min (\delta-1, \sigma-2)>0$.

Далее, используя соотношения (13), (29) и аналитичность функций $f_{1}, f_{2}$, получаем неравенство

$$
\left|f\left(k_{3}, \sqrt{k_{3}^{2}+A \epsilon^{\sigma}}\right)-f\left(\tilde{k}_{3}, \sqrt{\tilde{k}_{3}^{2}+A \epsilon^{\sigma}}\right)\right| \leqslant C_{2}\left|k_{3}-\tilde{k}_{3}\right| .
$$

Наконец, заметим, что функция $\beta_{k_{\|}}=\beta_{k_{\|}}\left(k_{3}, \epsilon\right)$ для достаточно малых $k_{3}$ и $\epsilon$ дифференцируема по $k_{3}$, причем

$$
\frac{d \beta_{k_{\|}}\left(k_{3}, \epsilon\right)}{d k_{3}}=O\left(\epsilon^{2}\right)
$$

Соотношения (28), (30)-(32) доказывают сжимаемость отображения $F$, а следовательно, существование единственного решения уравнения (22) (или, что то же, уравнения (14)) для всех достаточно малых $\epsilon$. Утверждения теоремы 1 вытекают из леммы 2 и равенства (26) (нужно возвести его в квадрат).

ЗАмЕчАНИЕ 1. Асимптотическая формула (21), в отличие от аналогичной формулы в следуюшей ниже теореме 2 , не является следствием того, что $k_{3} \in S_{\epsilon}$. По своей структуре формула (21) идентична формуле для $V_{0}=0$ [5] (если $\sigma \geqslant 3$, то $\nu=2$ и (21) совпадает с формулой для $V_{0}=0$ ), чего нельзя сказать о формуле из теоремы 2 , в которой скорость убывания величины ступеньки меньше.

ЗАмЕчАниЕ 2. Из равенств (3), (24) и предложения 3 вытекает, что в случае, если

$$
\int_{\Omega} W(x) d x<0
$$

уровень $E$ - это собственное значение, а в случае, если

$$
\int_{\Omega} W(x) d x>0
$$

уровень $E$ - резонанс. Аналогично в нижеследующей теореме $2 E$ - это собственное значение.

В теореме 2 в отличие от теоремы 1 предполагается, что $\sigma=2$, а константа $\delta$ заключается в промежутке $(1,2)$. По-прежнему число $K>0$ произвольно.

ТЕОРЕМА 2. Предположим, что выполнены условия

$$
\int_{\Omega} W(x) d x<0, \quad A<\frac{1}{2}\left(\int_{\Omega} W(x) d x\right)^{2} .
$$

Тогда уравнение (8) имеет при всех достаточно малых $\epsilon$ для совокупности чисел Е, удовлетворающих неравенству

$$
\left|\sqrt{E-k_{\|}^{2}}-\frac{\epsilon}{2 i}\left(\int_{\Omega} W(x) d x+\frac{A}{\int_{\Omega} W(x) d x}\right)\right| \leqslant K \epsilon^{\delta},
$$

единственное с точностью до множителя ненулевое решение в классе $L^{2}(\Omega)$. Для соответствующего уровня Е справедливо соотношение

$$
E=k_{\|}^{2}-\frac{\epsilon^{2}}{4}\left(\int_{\Omega} W(x) d x+\frac{A}{\int_{\Omega} W(x) d x}\right)^{2}+O\left(\epsilon^{1+\delta}\right) .
$$


ДОКАЗАТЕЛЬСТВО. Положим

$$
S_{\epsilon}^{\prime}=\left\{k_{3} \in \mathbb{C}:\left|k_{3}-\frac{\epsilon}{2 i} \frac{f^{2}(0,0)+A}{f(0,0)}\right| \leqslant K \epsilon^{\delta}\right\} .
$$

Для $k_{3} \in S_{\epsilon}^{\prime}$ имеем

$$
k_{3}=-\frac{i \epsilon}{2} \frac{f^{2}(0,0)+A}{f(0,0)}+\gamma\left(k_{3}, \epsilon\right),
$$

где $\left|\gamma\left(k_{3}, \epsilon\right)\right| \leqslant K \epsilon^{\delta}$. Уравнение (14) или, что то же, (22) будет рассматриваться в множестве $S_{\epsilon}^{\prime}$.

С помощью (35) и (33) найдем

$$
\sqrt{k_{3}^{2}+A \epsilon^{2}}=-\frac{i \epsilon}{2} \frac{f^{2}(0,0)-A}{f(0,0)}+\frac{f^{2}(0,0)+A}{f^{2}(0,0)-A} \gamma\left(k_{3}, \epsilon\right)+O\left(\epsilon^{2 \delta-1}\right) .
$$

Докажем, что (в обозначениях предыдущей теоремы) отображение $F$ переводит множество $S_{\epsilon}^{\prime}$ в себя. Пусть $k_{3} \in S_{\epsilon}^{\prime}$. Пользуясь соотношениями $(27),(35),(36)$ и $(25)$, находим

$$
\left|F\left(k_{3}\right)+\frac{i \epsilon}{2} \frac{f^{2}(0,0)+A}{f(0,0)}\right|=\frac{1}{2}\left|\frac{2 A}{f^{2}(0,0)-A} \gamma\left(k_{3}, \epsilon\right)+O\left(\epsilon^{2 \delta-1}\right)+O\left(\epsilon^{2}\right)\right| .
$$

Полученное выражение не превосходит $K \epsilon^{\delta}$ для достаточно малых $\epsilon$ при условии, что

$$
\left|\frac{A}{f^{2}(0,0)-A}\right|=\frac{A}{f^{2}(0,0)-A}<1 \text {. }
$$

Условие (37) выполнено в силу предположения (33).

Докажем сжимаемость отображения $F$. Используя первое равенство в (29), а также соотношения (35) и (36), имеем

$$
\frac{1}{2}\left|\left(k_{3}-\sqrt{k_{3}^{2}+A \epsilon^{2}}\right)-\left(\tilde{k}_{3}-\sqrt{\tilde{k}_{3}^{2}+A \epsilon^{2}}\right)\right|=\frac{\left|k_{3}-\tilde{k}_{3}\right|}{2}\left|\frac{-2 A+O\left(\epsilon^{\delta-1}\right)}{f^{2}(0,0)-A+O\left(\epsilon^{\delta-1}\right)}\right| .
$$

Далее, аналогично оценке (31) с помощью также (38) получаем

$$
\begin{aligned}
\mid f\left(k_{3},\right. & \left.\sqrt{k_{3}^{2}+A \epsilon^{2}}\right)-f\left(\tilde{k}_{3}, \sqrt{\tilde{k}_{3}^{2}+A \epsilon^{2}}\right) \mid \leqslant \\
& \leqslant C\left(\left|\sqrt{k_{3}^{2}+A \epsilon^{2}}-\sqrt{\tilde{k}_{3}^{2}+A \epsilon^{2}}\right|+\left|k_{3}-\tilde{k}_{3}\right|\right)= \\
& =C\left|k_{3}-\tilde{k}_{3}\right|\left(\left|\frac{f^{2}(0,0)+A+O\left(\epsilon^{\delta-1}\right)}{f^{2}(0,0)-A+O\left(\epsilon^{\delta-1}\right)}\right|+1\right) .
\end{aligned}
$$

Из соотношений $(28),(38),(39)$ и (31), а также из справедливости неравенства (37) следует сжимаемость отображения $F$. Тем самым сушествование требуемого решения уравнения (8) доказано. Асимптотическая формула (34) вытекает из (35). Теорема доказана.

Теоремы 1 и 2 при вьполнении некоторых условий исчерпьвают возможности получения простых асимптотических формул для решений $k_{3}$ уравнения (14) (соответственно для уровня $E$ уравнения (8)) в описываемой ситуашии. Более точно, предположим, 
что $V_{0}=-A \epsilon^{\sigma}$, где $A>0, \sigma \geqslant 1$ (см. следствие леммы 2). Рассмотрим возможность представления решения $k_{3}$ уравнения (14) (или (22)) в виде

$$
k_{3}=B \epsilon^{a}+O\left(\epsilon^{a+\mu}\right), \quad B \neq 0, \quad a, \mu>0 .
$$

Предположим, что выполнены соотношения

$$
\begin{aligned}
\frac{A}{2 B}+i f(0,0) & \neq 0, \\
A+B^{2} & \neq 0 .
\end{aligned}
$$

ТЕОРема 3. Пусть при всех достаточно мальх $\epsilon>0$ существует решение $k_{3}$ уравнения (22), для которого выполнены соотношения (40). Тогда для доста точно мальх $\epsilon$ либо $\sigma>2, a=1$ и $k_{3}$ совпадает с решением из формулировки теоремы 1 , либо $\sigma=2, a=1$; если в последнем случае $\mu>\delta-1$, где $\delta$ взято из формулировки теоремы 2, и выполнены условия (33), то $k_{3}$ для малых $\epsilon$ совпадает с решением, определяемым теоремой 2.

ДокАЗАТЕЛЬство. В силу равенства (22) имеем

$$
k_{3}=-\sqrt{k_{3}^{2}+A \epsilon^{\sigma}}+\frac{\epsilon}{i} f\left(k_{3}, \sqrt{k_{3}^{2}+A \epsilon^{\sigma}}\right)+O\left(\epsilon^{2}\right) .
$$

Пусть вначале $\sigma>2 a$. Тогда вследствие соотношений (40), (43) получаем

$$
k_{3}=-k_{3}+\phi\left(k_{3}, \epsilon\right)+\frac{\epsilon}{i} f(0,0)+O\left(\epsilon^{1+a}\right)+O\left(\epsilon^{2}\right)
$$

где

$$
\phi\left(k_{3}, \epsilon\right)=-\frac{A \epsilon^{\sigma}}{2 k_{3}}+o\left(\epsilon^{\sigma-a}\right)=-\frac{A \epsilon^{\sigma-a}}{2 B}+o\left(\epsilon^{\sigma-a}\right)=O\left(\epsilon^{\sigma-a}\right) .
$$

Если при этом $a>1$, то

$$
k_{3}=\frac{\epsilon}{2 i} f(0,0)+O\left(\epsilon^{\sigma-a}\right)+O\left(\epsilon^{2}\right)
$$

Сравнение (46) с (40) приводит к противоречию, т.к. $a=1$. Пусть $a<1$. Сравнивая (44) с (40) (учитывая при этом (45)), также легко приходим к противоречию (в случае $\sigma-1=1$ используем еще и условие (41)). Рассмотрим оставшуюся возможность $a=1$, тогда $\sigma>2$. Вследствие (44), (45) получаем

$$
\left|k_{3}-\frac{\epsilon}{2 i} f(0,0)\right| \leqslant C \epsilon^{\nu},
$$

где $\nu=\min (\sigma-1,2)$, так что для достаточно малых $\epsilon$ имеем $k_{3} \in S_{\epsilon}$ (определение множества $S_{\epsilon}$ см. в доказательстве теоремы 1$)$. Таким образом решение уравнения $(22)$ совпадает с решением (21) из теоремы 1.

Рассмотрим теперь случай $\sigma<2 a$. В силу соотношений (40) и (43) имеем

$$
k_{3}=-\sqrt{A} \epsilon^{\sigma / 2}+O\left(\epsilon^{2 a-\sigma / 2}\right)+\frac{\epsilon}{i} f(0,0)+O\left(\epsilon^{1+\sigma / 2}\right)+O\left(\epsilon^{2}\right) .
$$


Сравнение равенств (47) и (40) приводит к противоречию.

Рассмотрим оставшийся случай $\sigma=2 a$. Имеем согласно (40) и (42)

$$
\sqrt{k_{3}^{2}+A \epsilon^{\sigma}}=\sqrt{B^{2}+A} \epsilon^{a}+O\left(\epsilon^{a+\mu}\right) .
$$

Вследствие (43)

$$
k_{3}=-\sqrt{B^{2}+A} \epsilon^{a}+O\left(\epsilon^{a+\mu}\right)+\frac{\epsilon}{i} f(0,0)+O\left(\epsilon^{1+a}\right)+O\left(\epsilon^{2}\right) .
$$

Пусть $a>1$, тогда из сравнения (48) с (40) следует, что $a=1$ (противоречие). В случае $a<1$ в силу условия (42) имеем $B=-\sqrt{B^{2}+A}$, откуда $A=0$, что также невозможно. Наконец, если $a=1$, то $\sigma=2$ и

$$
k_{3}=\left(-\sqrt{B^{2}+A}+\frac{1}{i} f(0,0)\right) \epsilon+O\left(\epsilon^{1+\mu}\right)+O\left(\epsilon^{2}\right),
$$

откуда согласно (40)

$$
B=-\sqrt{B^{2}+A}+\frac{1}{i} f(0,0)
$$

Следовательно,

$$
B=\frac{A+f^{2}(0,0)}{2 i f(0,0)}
$$

В соответствии с (49)-(51)

$$
\left|k_{3}-\frac{A+f^{2}(0,0)}{2 i f(0,0)} \epsilon\right| \leqslant C \epsilon^{\rho},
$$

где $\rho=\min (1+\mu, 2)$. Если $\mu>\delta-1$, то $\rho>\delta$ и $k_{3} \in S_{\epsilon}^{\prime}$ для малых $\epsilon$ (определение $S_{\epsilon}^{\prime}$ см. в доказательстве теоремы 2). Теорема 3 доказана.

\section{Список литературы}

[1] Ю. П. Чубурин. ТМФ. 1988. Т. 77. № 3. С. 472.

[2] E. B. Davies. Proc. Cambridge Philos. Soc. 1977. V. 82. P. 327.

[3] E. B. Davies, B. Simon. Commun. Math. Phys. 1978. V. 63. P. 277.

[4] М. Рид, Б. Саймон. Методы современной математической физики. Т. 4. Анализ операторов. М.: Мир, 1982.

[5] Ю. П. Чубурин. Матем. заметки. 1992. Т. 52. № 2. С. 138.

[6] Ю.П. Чубурин. О рассеянии на кристаллической пленке (спектр и асимптотика волновых функций уравнения Шредингера). Препринт. Свердловск: Физико-технический институт УНЦ AH CCCP, 1985.

[7] Ю. П. Чубурин. ТМФ. 1987. Т. 72. № 1. С. 120.

[8] Х. Цикон, Р. Фрезе, В. Кири, Б. Саймон. Операторы Шредингера с приложениями к квантовой механике и глобальной геометрии. М.: Мир, 1990.

[9] Ю. П. Чубурин. ТМФ. 1997. Т. 110. № 3. С. 443.

[10] М. Рид, Б. Саймон. Методы современной математической физики. Т. 3. Теория рассеяния. М.: Мир, 1982. 